Thorax (1968), 23, 204.

\title{
Emergency oesophagectomy
}

\author{
W. F. K E R R ${ }^{1}$ \\ From the Thoracic Surgical Unit, Papworth Hospital, Cambridge
}

In the treatment of instrumental perforation of the obstructed thoracic oesophagus, relief of obstruction is one of the prerequisites of success. In some cases it is better to resect both the perforation and the original lesion rather than to rely on repair and drainage. The salient features of 19 cases of emergency oesophagectomy collected from the literature have been tabulated, and three new examples are here reported. The results are encouraging. The operation usually performed for malignant cases is a one-stage oesophagogastrectomy with oesophagogastrostomy ; a two-stage procedure is recommended for benign lower-end strictures. The lacerated oesophagus and the stricture are resected at the emergency operation, and the fundus of the stomach, advanced into the chest, is anastomosed to the oesophagus. The whole of the stomach is thereby preserved for the elective reconstruction which constitutes the second stage.

Instrumental perforation of the oesophagus, a dangerous complication of endoscopy, is easily diagnosed, but treatment is still controversial. This paper is concerned only with the treatment of perforation associated with chronic organic obstruction.

Most perforations occur as a result of foreignbody impaction in children and involve chiefly the pharynx or cervical oesophagus (Dawes, 1964). Diagnostic endoscopy in adults is responsible for the second largest group, and it is the cervical oesophagus that is most liable to injury. Recent papers have emphasized that the oesophagus is normal before the accident in the majority of patients, and opinions and statistics on treatment are coloured by this preponderance, to the detriment of the small but nevertheless important minority who have oesophageal disease. In them, perforation is most likely to occur during attempts to inspect, to take a biopsy, or to dilate chronic obstructive lesions of the thoracic or abdominal oesophagus, and, by contrast with the majority, the injury is almost always at or just above the upper limit of the obstruction.

There is agreement that any method of treatment stands its best chance of success if it is started early, but the importance of unrelieved obstruction distal to the perforation appears to have gone unremarked until Davidson (1965) and Groves (1966) were provoked to reply to a muchpublicized article (Mengoli and Klassen, 1965) which advocated non-operative management for

1Present address: Norfolk and Norwich Hospital, Norwich, NOR 53A all cases in the first instance. In the past, the subject has been confused by the practice of reporting together all examples of perforation seen in any one clinic, whether with or without obstruction and howsoever caused. No one before Groves (1966) appears to have analysed results primarily $\varrho$ by reference to the underlying pathology, though $\overrightarrow{\vec{O}}$ this would seem to be a factor of some importance. Hitherto, classification of oesophageal injuries had usually been according to site, and? sometimes also according to the method of production and size. The indications for instrumentation have not always been stated and the final diagnosis has frequently been omitted. Results, $\frac{\text { 의 }}{3}$ measured in terms of life and death, have been related mainly to the time that had elapsed before 0 active treatment was begun, and little attention has been paid to any other factor. This paper $ᄋ$ does not attempt to review all aspects of instru- $\rightarrow$ mental perforation but discusses treatment when the injury is associated with oesophageal obstruc- $N$ tion, and it is assumed that full supportive measures and antibiotics are exhibited efficiently. $\mathcal{O}$ I think that the effects of the original oesophageal $w$ lesion must be taken into account when treatment of an accidental perforation is being determined,o and $I$ here report three cases of emergency oeso- $\mathbb{\Phi}$ phagectomy carried out successfully in obedience? to this principle and discuss the problem of how much of the stomach should be resected when the stricture is benign.

When the diagnosis of intrathoracic perfora $-\frac{\mathbb{Q}}{\Omega}$ tion of the oesophagus is made within 12 to 180 hours of the accident, most thoracic surgeons, 
Mengoli and Klassen (1965) notwithstanding, would operate with the intention of repairing the damage and draining the pleura and mediastinum. Some would go no further even when faced with obstruction, despite the fact that a fistula will not heal and cannot be closed in such circumstances. They hold that resection is too hazardous. This is the orthodox approach if the view of Logan (1965) is representative. Distinguishing between obstructed and unobstructed cases only by implication, he advised against a procedure of the severity of oesophageal resection though he would permit, 'for example', repair of a hiatal hernia at the same time as closure of the perforation in a patient who is not gravely ill and for whom an effective antibiotic is available'. Terracol and Sweet (1958) treated perforations on similar lines but gave tacit approval to oesophagectomy in certain cases by adding that if the injury appears to be so extensive that resection may be necessary' a left-sided approach should be chosen. On the other hand, there is the impression that immediate resection in appropriate cases is already so widely practised as scarcely to deserve comment. It is recommended without discussion in books by Borrie (1959) and Flavell (1963) and supported by a recent annotation in the Lancet (1966). None of these authorities, for or against, backed his opinions by quoting gases within his own experience, but Borrie referred to an article by McBurney, Kirklin, Hood, and Andersen (1953) which described three cases of carcinoma of the lower oesophagus or cardia treated by resection 10 hours to five days after accidental perforation. Emergency oesophagectomy is the subject of at least two other articles in American journals (Satinsky and Kron, 1952 ; Blalock, 1957), while Groves (1966) gives examples and discusses the problem in the larger context of oesophageal perforations in general.

T A B L E

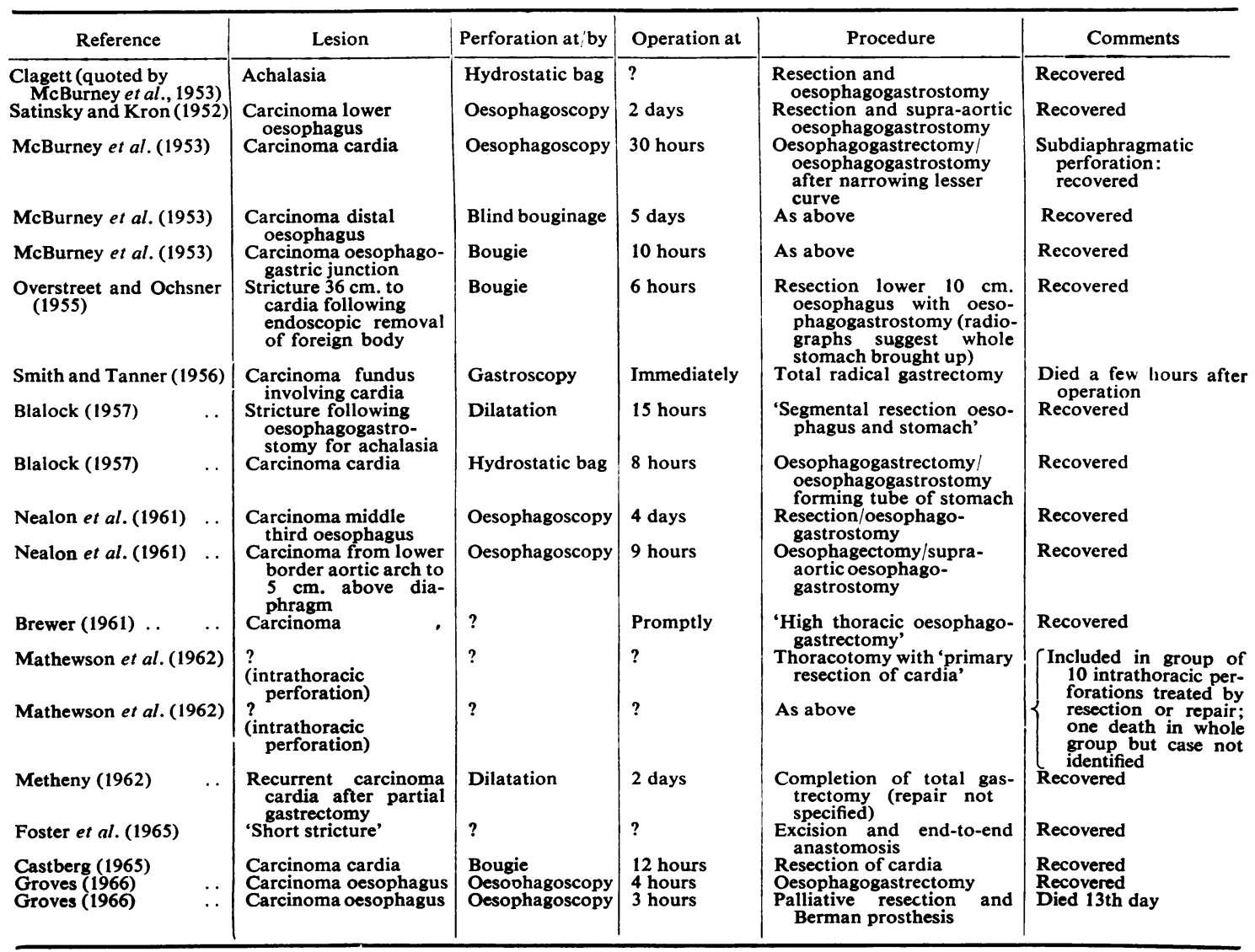


Clagett in 1946 or 1947 was probably the first to carry out successfully an emergency oesophagectomy and oesophagogastrostomy. The patient suffered from achalasia, and the oesophagus had been ruptured with a hydrostatic bag. This case has not been reported in full but is referred to by the Mayo Clinic group (McBurney et al., 1953, vide supra), who were second in the field with published results. First in print were Satinsky and Kron (1952) with a single case operated on in 1949, a carcinoma resected two days after perforation of the oesophagus at oesophagoscopy. Reconstruction was by supraaortic oesophagogastrostomy. There was no postoperative obstruction or leakage; the patient was in hospital for six weeks and was well two years later. The Table summarizes 19 cases of emergency resection from the literature. In addition there are references, generally brief and lacking in detail, to eight cases of achalasia treated by Heller's operation at the same time as closure of the laceration (Barrett, 1956-2 cases ; Dawes, 1964-1 case ; Foster, Jolly, Sawyers, and Daniel, 1965-3 cases; Groves, 1966-2 cases) and two of simultaneous repair of a hiatal hernia (Castberg, 1965 ; Groves, 1966). All these patients made good recoveries. One fatal case of benign cicatricial stenosis treated by 'Heller-type myotomy' and repair of hiatal hernia (Nealon, Temple- ton, Cuddy, and Gibbon, 1961) is not included in this list as the perforation was neither repaired? nor resected.

\section{CASE REPORTS}

CASE 1 H. W., a man of 50 years, had a long benigñ stricture of the oesophagus of unknown aetiology $\overrightarrow{0}$ He gave a history of having eaten when a boy somesweets that had been dipped in acid. He had sufferect no ill effects at the time. An unrelated condition was cardiomegaly from aortic incompetence. In Apri 1965 he developed for the third time complete oesoi phageal obstruction from impaction of a piece of meat at the lower end of the stricture. Fibrosis pre N vented the oesophagoscope being passed beyond mid요 thoracic level, but the piece of meat could just be seen, and much of it was removed with grasping forceps. The patient made no complaints after this manipulation, in fact he said his swallowing wa improved. Two days later it was clear that, in spitक్ of his protestations, he still suffered from almost com plete obstruction, and a Gastrografin swallow waso ordered. It showed the oesophagus to be stilgo obstructed by impacted food. a perforation imme: diately above the obstruction, and an abscess in the mediastinum (Fig. 1).

Operation (48 hours after injury) Through a leff̆ thoracotomy the oesophagus was mobilized and the small mediastinal abscess was opened and evacuated The perforation was situated in the right wall of the
FIG. 1. Case 1. Gastrografin swallow 45 hours after endoscopy showing the long strictured segment perforated at its lower end just above the diaphragm.

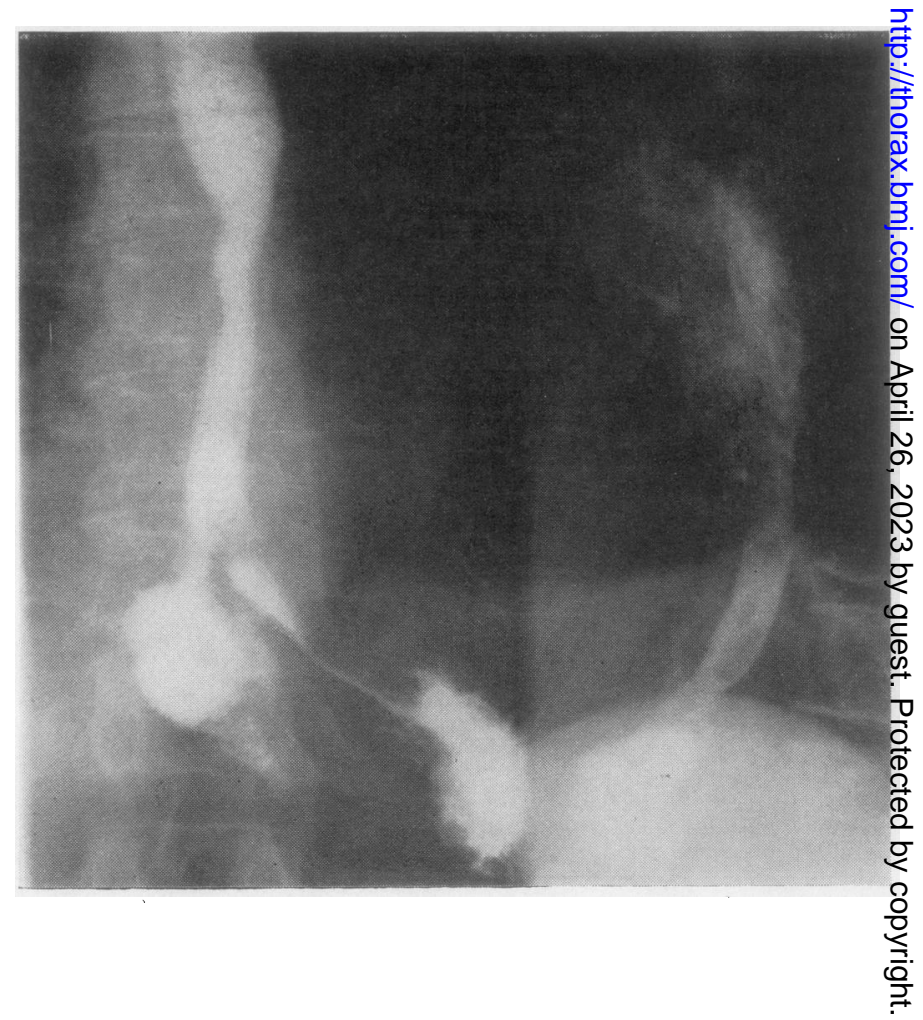


oesophagus in an inaccessible position just above the narrowest and most fibrotic part. The diaphragm was incised radially to the hiatus and the lower end of the oesophagus was detached from the stomach. The segment containing the stricture, the piece of meat, and the perforation was resected, dividing the oesophagus again at the level of the inferior pulmonary vein where its wall was fibrotic and inelastic. After closing the cardiac orifice the stomach was mobilized by removing the spleen and dividing the left gastric artery, and a pyloromyotomy was carried out. The oesophagus was then anastomosed to the fundus of the stomach. The patient made an uneventful recovery and was discharged.

$\mathrm{He}$ was reluctant to submit to another operation but did so after eight months had passed. By then he had had several dilatations for dysphagia and had suffered much oesophageal pain that closely mimicked myocardial ischaemia. At the second operation the stomach was mobilized and replaced in its normal position below the diaphragm with an isolated jejunal loop as substitute for the resected oesophagus. The patient again made a satisfactory recovery, complaining only of colicky postprandial pain for the first month or so. This yielded to antispasmodics.

CASE 2 H. G., aged 74 years, had severe chronic bronchitis and gout in addition to dysphagia from a

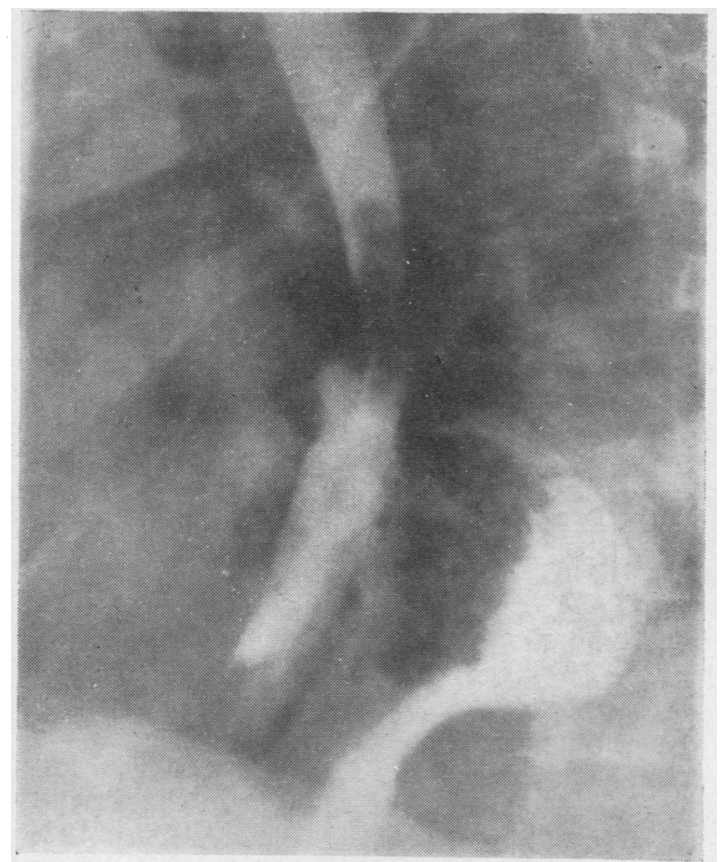

FIG. 2. Case 2. Gastrografin swallow six hours after attempted dilatation of a peptic stricture. The oesophagus was perforated just above the stricture and the false passage lies in front (and to the right) of the recurrent hiatal hernia. peptic stricture of the oesophagus. Three years earlier the stricture had been dilated and a hiatal hernia repaired. Dysphagia recurred 18 months after the operation, and in the succeeding 15 months six dilatations of the stricture were required. It was after the last of these that he complained of severe precordial pain and was found to be sweating profusely. A perforation immediately above the stricture was demonstrated radiologically (Fig. 2).

Operation (eight hours after perforation) Exactly the same procedure was carried out as in the first stage of case 1 . The perforation was in the right side of the oesophagus and difficult of access. The recent history indicated that dilatation was not effectively treating the obstruction, and resection was imperative.

The post-operative course was stormy. He developed atrial fibrillation, hypotension, oliguria, lethargy, and sputum retention, but eventually made a slow, complete recovery. There has been no recurrence of dysphagia. A barium swallow in April 1966, nine months after the operation, showed a good lumen at the anastomosis and no delay in the passage of barium.

CASE 3 L.T., a frail woman of 68 years, presented with symptoms of complete oesophageal obstruction. A barium swallow showed a mid-oesophageal stricture that could equally well have been benign or malignant. At oesophagoscopy this very tight stricture was dilated and a biopsy was taken, after which the lung was seen and this indicated a rupture of the oesophagus. The patient was immediately turned into the right lateral position and the left side of the chest was opened. The stricture, which was no more than $1 \mathrm{~cm}$. in length, was surrounded by much fibrosis, and the nature of the obstruction remained obscure. After the lower segment had been opened and had been seen to be lined with gastric-type epithelium, a local resection was carried out, and the patient made an excellent recovery. Histological examination of the resected specimen confirmed that the lower segment was lined by columnar epithelium, but showed squamous carcinoma in the stricture and in an adjacent lymph node less than $5 \mathrm{~mm}$. in diameter. Six months after the operation carcinoma recurred in the suture line and a Souttar's tube was inserted.

\section{COMMENT}

These three cases and the 19 collected from the literature make a total of 22 emergency resections for instrumental perforation of the oesophagus associated with chronic obstruction. With the majority of patients making rapid and uncomplicated recoveries and only two deaths (possibly three if the fatal case in the series of Mathewson, Dozier, Hamill, and Smith (1962) was one of 
their two resections) the results compare favourably with those of elective oesophagectomy and represent an improvement on other methods of treating perforations. But such a comparison is invalid. On the one hand, most published series of perforations include cases beyond all hope when first seen by a competent oesophageal surgeon, and, on the other, the contribution of persistent obstruction to mortality and morbidity has never been separately catalogued. On general principles obstruction would seem to be one of the most significant factors, and the cases treated by resection might very well be considered those with the worst prognosis. What the results show is that emergency oesophagectomy can succeed even in the presence of purulent mediastinitis. It should therefore be given consideration when less radical methods offer only slender prospects of recovery.

\section{DISCUSSION}

Oesophagectomy is a formidable operation at the best of times, and certainly not one to be undertaken lightly when conditions, both general and local, are unfavourable. The normal oesophagus is poor material for suturing. After it has been traumatized and infected for some hours even the most optimistic surgeon would not care 'to place much reliance on the security of his suture line. Repair, when there is no obstruction, holds prospects of success only if accomplished within 12 to 18 hours of the accident, and even then breakdown is frequent and generally fatal. Slight or partial obstruction does not preclude oesophageal healing, but severe obstruction distal to the perforation greatly increases the likelihood of failure. In a patient suffering from incurable cancer heroic measures are out of place, but if the original disease is not necessarily fatal, resection of the lesion together with the perforation may be the most hopeful approach. It allows anastomosis of the stomach to comparatively normal oesophagus and at the same time removes one of the most important factors militating against successful closure. The only alternative is to try to establish a fistula from the oesophagus across the pleura to the intercostal drain, attempting all the while to keep the lung expanded, the empyema localized, and the patient nourished (Froggatt and Gunning, 1966). Not surprisingly, the few survivors show little enthusiasm for still more surgery directed against the original complaint, and this is sometimes used as an argument in favour of immediate resection. A better reason is that the chances of recovery from the accident of perforation are increased rather than dimin- $\frac{\overrightarrow{\vec{P}}}{\vec{P}}$ ished by eliminating obstruction. The question the surgeon should ask when faced with this situa- $\frac{\bar{\sigma}}{\frac{5}{5}}$ tion is not so much whether the patient can stand $\overrightarrow{\widetilde{\sigma}}$ an oesophagectomy as whether he can stand an $\varrho$ oesophago-pleural fistula, which is what a repair in the circumstances amounts to. And until the $\overrightarrow{0}$ chest has been opened it is too early to decide that the patient is unfit for any more lengthy or $\vec{\omega}$ intricate procedure. The collapse associated with $\stackrel{\circ}{\rightleftharpoons}$ large perforations resists ordinary resuscitative $\vec{x}$ measures, and is reversible only after the pleura $\underset{\omega}{N}$ and mediastinum have been cleaned out.

It might be appropriate to mention a procedure $\tilde{O}$ theoretically applicable to certain benign cases though it does not appear to have been used as yet in the circumstances under discussion. The $\vec{z}$ recently described fundal patch operation (Thal and Hatafuku, 1964 ; Thal, Hatafuku, and Kurtz- $\frac{\widehat{S}}{9}$ man, 1965) has already been used successfully to $\vec{\odot}$ close a spontaneous perforation of the oesophagus $\mathscr{\odot}$ and also as a cardioplasty for the relief of benign lower-end stricture. It should be possible to combine these two functions by enlarging a traumatic perforation downwards through the stricture before applying the patch. To be suitable for this the perforation would have to be through the $\stackrel{\mathbb{Q}}{\propto}$ front or left side of the oesophagus, whereas pre- $\overrightarrow{\overrightarrow{0}}$ sent experience indicates that, as likely as not, 3 the inaccessible right side is affected. In a favourable case this operation could do away with the need for resection and would involve little more than simple suture of the oesophagus.

Given the decision to resect, how much should $\stackrel{0}{x}$ be removed and what form of reconstruction is best ? With two exceptions (Smith and Tanner, 1956; Groves, 1966) each case of malignant $\frac{\delta}{3}$ disease listed in the Table was treated by formal oesophagogastrectomy with oesophagogastros- $\frac{9}{2}$ tomy. Few will quarrel with this, for a malignant $D$ lesion is rarely suitable for local excision, and any other type of reconstruction adds very consider- N ably to the length and magnitude of the operation. A different approach is called for when the $N$ stricture is benign, and situated, as it so often is, $\underset{\omega}{ }$ at the oesophago-gastric junction. It seems wrong $\bar{\sigma}$ to sacrifice more than half of a healthy stomach 0 to achieve a reconstruction that is far from ideal and quite likely to need revision in the years to $\stackrel{?}{+}$ come, a revision moreover that would have to start with the handicap imposed by a gastric resection. It is inviting another stricture to anastomose the fundus of the stomach to the oesophagus $\stackrel{\mathbb{Q}}{\square}$ in the chest (Barrett and Franklin, 1949), but the operation is quick and easy, the patient will be no 
worse off than before the accident, and the whole of his stomach will be available for whatever reconstruction may be thought best when conditions are more favourable. In case 1 both stages were completed, and the result is satisfactory so far. In case 2 the elderly and ailing patient understandably refused the second operation, but it could still be carried out should it become essential. On the other hand, the temporary repair may well last him the rest of his life.

Grateful acknowledgement is made to Drs. G. I. Verney and J. Rymer for the radiological investigations, and to Mr. E. W. Groves for reproducing the radiographs.

\section{REFERENCES}

Barrett, N. R. (1956). Perforations of the oesophagus and of the pharynx. Proc. roy. Soc. Med., 49, 529.

- - and Franklin, R. H. (1949). Concerning the unfavourable late results of certain operations performed in the treatment of cardiospasm. Brit. J. Surg., 37, 194.

Blalock, J. (1957). Primary esophagogastrectomy for instrumental perforation of the esophagus. Amer. J. Surg., 94, 393.

Borrie, J. (1959). The Management of Emergencies in Thoracic Surgery, p. 278. Staples Press, London.

Rrewer, L. A. (1961). Discussion of Nealon et al. (1961). J. thorac. cardiovasc. Surg., 41, 102.

Castberg, T. (1965). Traumatic perforation of the oesophagus. Acta chir. scand., Suppl. 343, p. 56.

Clagett, O. T. Quoted by McBurney et al. (1953).

Davidson, J. S. (1965). In Medical Forum. Mod. Med. G.B., 10, 959.

Dwesa, J. D. K. (1964). Traumatic perforations of the pharynx and oesophagus. J. Laryng., 78, 18.
Flavell, G. (1963). The Oesophagus, p. 71. Butterworths, London.

Foster, J. H., Jolly, P. C., Sawyers, J. L., and Daniel, R. A. (1965). Esophageal perforation: diagnosis and treatment. Ann. Surg., 161,701.

Froggatt, D. L., and Gunning, A. J. (1966). Treatment of oesophageal perforation. Thorax, 21, 524.

Groves, L. K. (1966). Instrumental perforation of the esophagus: What is conservative management? J. thorac. cardiovasc. Surg., 52, 1.

Lancet (1966). Annotation: oesophageal perforation, 2, 788.

Logan, A. (1965). Mediastinum. In Thorax, ed. d'Abreu, A. L. (Vol. 5 of Clinical Surgery, ed. Rob, C., and Smith, R.), Ch. 15, p. 217. Butterworths, London.

Mathewson. C., Dozier, W. E., Hamill, J. P., and Smith, M. (1962). Clinical experiences with perforation of the esophagus. Amer.J. Surg., 104, 257.

McBurney, R. P., Kirklin, J. W., Hood, R. T., and Andersen, H. A. (1953). One-stage esophagogastrectomy for perforated carcinoma in the presence of mediastinitis. Proc. Mayo Clin., 28, 281.

Mengoli, L. R., and Klassen, K. P. (1965). Conservative management of esophageal perforation. Arch. Surg., 91, 238.

Metheny, D. (1962). Discussion of Mathewson et al. (1962). Amer. J. Surg., 104, 265.

Nealon, T. F., Templeton, J. Y., Cuddy, V. D., and Gibbon, J. H. (1961). Instrumental perforation of the esophagus. J. thorac. cardiovasc. Surg., $41,75$.

Overstreet, J. W., and Ochsner, A. (1955). Traumatic rupture of the

to esophagus. J. thorac. Surg., 30, 164.

Satinsky, V. P., and Kron, S. D. (1952). One-stage esophagectomy in presence of mediastinitis. Arch. Surg., 64, 124.

Smith, C. C. K., and Tanner, N. C. (1956). The complications of gastroscopy and oesophagoscopy. Brit. J. Surg., 43, 396.

Terracol, J., and Sweet, R. H. (1958). Diseases of the Esophagus, Ch. 23, p. 443. Saunders, Philadelphia and London.

Thal, A. P., and Hatafuku, T. (1964). Improved operation for esophageal rupture. J. Amer. med. Ass., 188, 826.

_- - and Kurtzman, R. (1965). A new method for reconstruction of the esophagogastric junction. Surg. Gynec. Obstet., 120, 1225 . 